\title{
A REVIEW ON INDOOR LOCALIZATION WITH INTERNET OF THINGS
}

\author{
C.Basri ${ }^{1}$, A.Elkhadimi ${ }^{2}$ \\ ${ }^{1}$ Dept of Telecomunication Systems, National Institute of Posts and Telecommunications (INPT), Rabat, Morocco, \\ basri@inpt.ac.ma \\ ${ }^{2}$ Dept of Telecomunication Systems, National Institute of Posts and Telecommunications (INPT), Rabat, Morocco, \\ elkhadimi@inpt.ac.ma
}

KEY WORDS: Indoor localization, Internet of Things (IoT), Location services, Indoor technologies, Inertial sensors, Wireless positioning

\begin{abstract}
:
The advancement of Internet of things (IoT) has revolutionized the field of telecommunication opening the door for interesting applications such as smart cities, resources management, logistics and transportation, wearables and connected healthcare. The emergence of IoT in multiple sectors has enabled the requirement for an accurate real time location information. Location-based services are actually, due to development of networks, sensors, wireless communications and machine learning algorithms, able to collect and transmit data in order to determine the target positions, and support the needs imposed by several applications and use cases. The performance of an indoor positioning system in IoT networks depends on the technical implementation, network architecture, the deployed technology, techniques and algorithms of positioning. This paper highlights the importance of indoor localization in internet of things applications, gives a comprehensive review of indoor positioning techniques and methods implemented in IoT networks, and provides a detailed analysis on recent advances in this field.
\end{abstract}

\section{INTRODUCTION}

The position information is an essential component of internet of things applications in indoor and outdoor environments. For outdoor environments location is obtained by GPS (Global Positioning System) or similar GNNS constellations, but to determine location of people and objects inside a closer structure, GPS does not work due to the lack of the sight line to satellite, and other obstacles such as the high attenuation of signals, multipath and noise interferences. (Basri and Elkhadimi, 2016) Therefore, in order to get accurate position an indoor positioning system is conceived to overcome these limitations.

Location Based Services are among the most attractive applications related to the IoT, and will serve as the first element of information context in all future applications related to "intelligent environments". The IoT network comprises a wide variety of technologies and devices incorporating sensors, which observe and measure the state of the real world, and actuators which act on it. The implemented devices has the ability to collect, manage and exchange data in order to estimate location information of target objects. On the other hand multiple requirements must be considered while designing an IoT positioning system such as precision, accuracy, complexity, scalability, cost and privacy.

Indoor localization systems are based on different positioning approaches, different techniques, and different technologies, which make them convenient for some applications and inconvenient for other applications. It's necessary to distinguish the advantages and disadvantages of indoor localization technologies, so that we can apply suitable technology to the right situation.

We aim to provide the reader with the necessary references to acquire the knowledge on positioning process, and to understand the fundamentals of indoor localization systems in internet of things.
This article provides an overview of all the elements of Indoor localization in IoT system: domains application, multitechnology gateways between objects and networks, telecommunications infrastructures dedicated to IoT and system performance parameters. On the other hand the analysed processes offer the reader new research challenges to be investigated in the future.

\section{INDOOR LOCALIZATION APPLICATIONS FOR IOT}

The fields of application of Internet of Things concepts and technologies are numerous, the most advanced are Smart cities, industry, agriculture, management, healthcare, assistant living and many other applications domains where IoT is making process better and simpler.

\subsection{Smart Cities:}

One of the major IoT domains is smart cities. In smart cities IoT is used to collect environmental and behavioural data to enable better monitoring, analysis and understanding of urban flows in order to improve services quality and way of life and offering more comfort and safety.

Modern cities have great numbers of indoor spaces and require constant caring processes and resources management. IoT location services can provide an intelligent management of energy, electricity, lighting distribution and consumption under heterogeneous circumstances, and integrate the position of devices with other data to provide more added values to the user. For example, collecting information on car traffic and mobility of people to optimize driving and walking spaces. Indeed, these data make it possible to adapt urban signage, improve mobility and transportation. Thus, the community can measure noise, pollutant, and light levels to adjust lighting and cleaning services. (Nasiri et al., 2019) 


\subsection{Smart Industry:}

This category includes the measurement of indoor air quality, monitoring the levels of toxic gases and oxygen inside chemical plants to ensure the safety of workers, property, and locating resources using passive and active tags.

The connected industry through the deployment of sensors and connected industrial objects, allows better knowledge of industrial systems and trains high productivity through efficient preventive maintenance, greater responsiveness and cost reduction.

\subsection{Smart Healthcare:}

IoT and medical devices can collect data, enable remote care and give patients more control on their treatment. Location services in IoT allows faster reactivity and better understanding of different behaviours through a local and accurate data sensing, monitoring of single people, assistance to elderly or disabled people living independently and tracking of patient inside hospitals (Hossain et al, 2019).

\subsection{Smart Agriculture:}

Smart agriculture based on IoT and location services aims to a better resource management and enhanced productivity in indoor farming and indoor urban agriculture spaces. The applied Wireless sensors networks form an acquisition platform that can realize large-scale complex monitoring and tracking tasks in agricultural fields.

\subsection{Smart Logistics and transportation:}

IoT in logistics and transportation helps manage efficiently supply chain like the tracking of connected objects and vehicles. This category includes the monitoring of sensitive goods such as jewellery, medicines or dangerous goods, the detection of storage incompatibility when flammable products are stored close to others containing explosive materials, the search for individual items in large areas such as warehouses or ports and monitoring shipping conditions quality for insurance and maintenance purposes.

\subsection{Human safety:}

Whenever technology is mentioned people assumes that the purpose is to enhance productivity and improve communication. However, technology for the purpose of human safety is getting more importance and become one of the most targeted topic for the researches. Various types of Human Safety Devices (HSD) are being developed and applied in numerous fields of application. (Sharma and Londhe, 2018) An essential functions of these devices are anomaly detection in critical systems, assistance in medical technology, driverless vehicles, smart PPE (personal protective equipment) that assesses and prevents danger for work-related incidents and injuries, by using variety of sensors and detection methods, so alerts and data can be sent to safety personnel and managers to get full visibility with advanced remote viewing capabilities.

Guidance and evacuation in emergency situations. Users of an indoor localization application can be notified of an emergency situation and directed to emergency exists using the best accessible routes relative to their positioning.

\subsection{Indoor Localization and Covid-19 Pandemic:}

The World Health Organization has declared COVID-19 as a pandemic and almost all countries across the globe have been affected by the virus both socially and economically.

In several countries around the world, states have put in place various surveillance solutions to follow and control the evolution of the coronavirus epidemic and help public health authorities track the spread of the decease. Researchers have exploited the use of indoor localization technologies, and wireless communications to provide solutions applied in multiple scenarios (Saeed et al., 2020). In social distancing for example, the implemented tags alerted users when they are too close to each other through sound or vibrations.

Other systems were developed to enhance disinfection of working environment, detection of people affected by the virus, prevention of bottlenecks \& crowds, and to ensure monitoring of people with Covid-19 in order to trace their paths and prevent the risk of infection (Kamal et al., 2020).

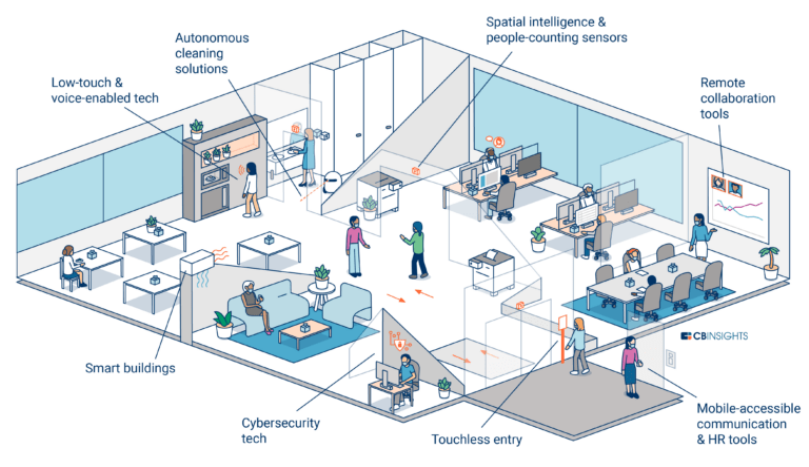

Figure1. IoT Applications to face Covid-19

\section{INDOOR LOCALIZATION TECHNOLOGIES FOR IOT}

Many developed technologies are implemented for ILBS (Indoor Location Based Services) have been studied, categorized mainly into infrastructure-based that requires pre-installed hardware such as Radio Frequency Identification (RFID), Infrared Radiation (IR), Bluetooth, Ultra-Wide Band (UWB),etc. and infrastructure-free that utilizes the existing resources that are already available in the environment, such as light-emitting diode (LED) and inertial sensors, these solutions are more attractive due to the low cost and ready-to-deploy characteristics.

Internet of things encompass multiple technologies explained in the following subsections.

\subsection{Localization technologies for IoT:}

\subsubsection{Wi-Fi}

Indoor location via Wi-Fi relies on the existing public or private Wi-Fi network. WiFi hotspots can be used to locate a smartphone or all kinds of Wi-Fi enabled devices. It is possible to determine the position of a user in a defined space, by measuring the intensity of the received signal, declaring reference points on map, then exploiting the use of mathematical models to match the recorded database with real measurements. This positioning process defined by fingerprinting method that will be explained in the following section.

The main limitation of Wi-Fi fingerprint technology is the timeconsuming database construction process, a lot of human intervention and constant environment changes. This drawbacks can be mitigated using machine learning algorithms for 
intelligent classification of indoor spaces, automatic and continuous radio map Update, sensors fusion and interaction of Wi-Fi infrastructure with others technologies (Yu et all., 2019).

\subsubsection{Inertial Sensors/ dead reckoning:}

Inertial sensors are based on the micro-electro-mechanical system (MEMS), which is a combination of micro-sensors, such as accelerometer, gyroscopes, magnetic compasses, etc. Pedestrian Dead Reckoning (PDR) is a method based on the inertial sensors which uses the number of steps that pedestrians walk to gain the results. Due to its high performances, it has become an area of interest in the positioning technology field.

An accelerometer measures changes in gravitational acceleration. It is usually used to measure accelerations, tilt and vibrations in numerous devices. Accelerometer measurement are represented by the acceleration component of the three-dimensional direction.

The gyroscope measures changes in orientation (angular movement), changes in rotational speed and provides the angular velocity. The magnetometer measures the strength and the direction of the magnetic field. (Kok et al., 2017)

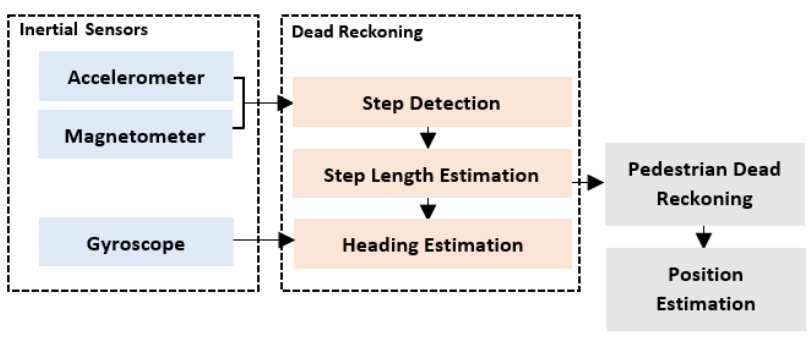

Figure 2. Pedestrian Dead Reckoning principle

The pedestrian's movement behaviour includes the distance and the movement direction. When a step has been detected, the position of that position can be calculated as:

$$
\begin{aligned}
& X_{t+l}=x_{t}+d \cos \left(r_{i}\right) \\
& Y_{t+l}=y_{t}+d \sin \left(r_{i}\right)
\end{aligned}
$$

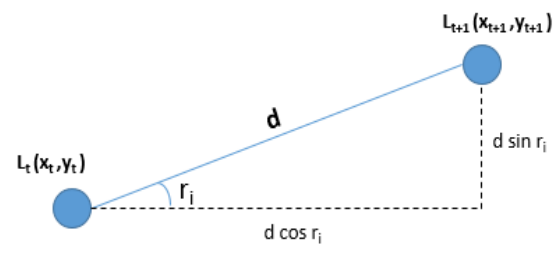

Figure 3. Position calculation for inertial navigation

Where: $\mathrm{x}_{\mathrm{t}}$ and $\mathrm{y}_{\mathrm{t}}$ are the coordinates in the two-dimensional coordinate system, $\mathrm{d}$ represents the step length of the user. The angle $r_{i}$ is obtained by combining data from the accelerometer, gyroscope and magnetometer.

\subsubsection{Infrared:}

Infrared (IR) are light signals invisible to the human eye because of longer wavelength to that of visible light. IR technology is widely used today in home automation and robotics. Examples of everyday applications are remote control (remote control) and obstacle detection. This technology has the advantage of being simple and inexpensive. The localization use can be divided into two approaches. In the first, called active, units made up of light- emitting diodes (LEDs) emit IR signals at regular intervals. These signals are decoded by light sensors, such as photodiode or IR camera, to provide information on the location of sources. In the second approach, called passive, the localization is carried out by thermography thanks to the emission of natural radiation from the entities to be located. IR signals are known to be sensitive to ambient light sources. Other disadvantages major aspects of IR technology are a constraint of line-of-sight between emitter and receiver as well as a range limited to 5-10 m typical.

\subsubsection{RFID}

Inexpensive and not requiring energy storage, they can be integrated into multiple objects that need to be identified and located. By transmitting a low-power radio frequency signal through its antenna, the tag reader supplies the tag's electronic chip with sufficient energy for the tag to retransmit through its own antenna a modulated signal representing the numeric identifier of the tag.

There are two possible ways for providing location information with RFID technology. A first consists in spreading a multitude of passive tags in the environment and fixing a reader on a device or on the user in order to locate the reader in relation to the tags. In a second more conventional use, fixed readers are arranged in critical locations in the environment and the tags are integrated into mobile devices or worn by the user in order to be located. In this solution it is possible to use more powerful readers, potentially with large antennas, so the range can be of the order of one meter.

\subsubsection{Ultrasound}

Ultrasound (US) are acoustic vibrations that extend from the border with audible acoustics to the thermal stirring frequencies of molecules around $1013 \mathrm{~Hz}$. Ultrasonic signals are usually generated by transducers that exploit the piezoelectric effect to convert electrical energy in mechanical movement and vice versa. Ultrasound based locating systems mainly operate in the frequency range $20-50 \mathrm{KHz}$. These signals have the advantage of being relatively slow to propagate, making it easier to take action temporal for this kind of application and improves location accuracy. The obtained results are often associated with very high precision, which may be less than a millimetre. However, by the nature of these signals, the range of the systems which use them is generally low, limited to $10-15 \mathrm{~m}$ typical. On the other hand, performance is affected by environmental conditions, such as temperature and humidity and drafts.

\subsubsection{Ultra Large Band}

Ultra Large Band (ULB) technology is based on the transmission of pulses of very short duration. Employment of ULB signals facilitate the detection of multipath thanks to the broadband property of these signals. The range varies from 10 to 100 meters depending on the sensors used. Two localization approaches are possible: active and passive. During the premiere, a ULB receiver extracts location information through ULB signals sent by transmitting entities. In order to avoid infrastructure dedicated to localization, the second approach - similar to radar mode, is based on a single ULB equipment, which emits a ULB signal. At reception, this equipment exploits, thanks to its antenna network, the use of affected signals by multipath in order to extract location data. On the other hand, beyond additional cost, the use of a receiver with an antenna network in terminals widespread in the large audience is very complex.

\subsubsection{Hybrid Systems}

Hybrid architectures are based on the interaction between different technologies and combine multiples sensors that overcome each other's drawbacks to make a more accurate system (De Angelis et al., 2015). 
The recent trend in indoor localization is to use hybrid system for locating the user position with minimum errors. Combining several types of technologies may be more advantageous as we can exploit the strengths of each technology simultaneously. Multiple localization fingerprint are combined with the help of sensor fusion framework. The most prominent hybrid localization system is the fusion of smartphone sensors and WiFi infrastructure (Li et al., 2016; Ebner et al., 2017; Yu et al., 2019).

\subsubsection{Comparative Study}

Most of existing tracking systems use infrared (IR), ultrasound (US), radio frequency (RF) and inertial sensor technologies. IRbased systems are limited to a location in a specific area but suffer from errors in obstructed spaces, and their performance is affected by ambient light sources. US systems provide very high accuracy using the time of flight signals. Unfortunately, they require special attention in receptors orientation. An alternative to IR and US technologies is carried by RF technologies. RFID systems have a short range but require a large number of tags.

The performance of systems using ZigBee, Bluetooth or WiFi technology, is affected by the presence of obstacles and multipath. Another possibility is given with the inertial sensors integrated on most current mobile terminals in order to set up a pedestrian dead reckoning system (PDR). NPE systems suffer from the accumulation of estimation errors. Other systems combine multiple technologies to take advantage of the benefits of each technology and improve system performance.

\subsection{Metrics of localization in IoT:}

The localization in IoT relies on different techniques, where measurements can be extracted from multiple domains classified mainly into three domains: Power or signal strength-based, Timebased and Space-based (Turgut et al., 2016).

\subsubsection{Power Domain}

For a signal from a transmitter, RSS (Received Signal Strength) is a measure of the received signal strength by a receiver. The basic idea exploits the relation of Friis (Friis, 1946), also called the "telecommunications equation" for free space propagation. In general, free space propagation conditions are rarely encountered. Several models have been proposed to model the power loss as a function of the distance between a transmitter and a radio receiver.

In indoor environment, the log-distance model is preferred. A simple form of this model is given by:

$$
\operatorname{Pr}(\mathrm{d})=\operatorname{Pr}(\mathrm{d} 0)-10 \alpha \mathrm{RSS} \log (\mathrm{d} / \mathrm{d} 0)
$$

Where $\operatorname{Pr}(\mathrm{d})$ is the power of received signal (in $\mathrm{dBm}$ ) at the distance $\mathrm{d}, \mathrm{d} 0$ a reference distance and $\alpha \mathrm{RSS}$ the propagation coefficient. The value of this coefficient is typically between 1.8 and 3.2 in indoor environments for signals produced in the frequency band $900 \mathrm{MHz}$ to $4 \mathrm{GHz}$. The model (2) can be improved by introducing additional corrective terms to take into account in particular the attenuation caused by multiple floors, walls. These terms, which depend on the materials, are determined empirically using calibration measurements.

The RSSI parameter has been used in vast indoor localization systems, however it suffers from performance degradation in complex environment due to interferences and multipath problems. To overcome these limits, Kalman filters are implemented on the positioning estimation process to improve location accuracy (Chen et al., 2015). On the other hand the convergence to hybrid infrastructure based on the interaction between RSSI and other RF metrics always provides better performance (Mendoza-Silva et al., 2019; Sadowski and Spachos, 2018).

\subsubsection{Time Domain}

Time measurements exploit the time propagation of the signal, also named ToF (Time of Flight), to estimate the distance between two different nodes A and B. The distance estimation is based on the following proportionality relationship:

$$
\mathrm{d}=\mathrm{c} \tau
$$

Where $\mathrm{d}$ is the distance travelled by the signal, $\tau$ the time of flight, and $\mathrm{c}$ the speed of the wave.

The best-known measurements in this category are TOA (Time Of Arrival), TDOA (Time Difference Of Arrival) and RTT (Round Trip Time).

\subsubsection{Space Domain}

Space domain includes angle (or direction) measurements. The main existing orientation measures are the Angle of Arrival (AOA) and the Phase Of Arrival (POA).

A summary of the metrics introduced in this section is given

\begin{tabular}{|c|c|c|}
\hline Metric & Principle & $\begin{array}{c}\text { Advantage (s) } \\
\text { /Inconvenient(s) }\end{array}$ \\
\hline TOA & $\begin{array}{l}\text { Distance } \\
\text { estimation based } \\
\text { on signal } \\
\text { propagation time }\end{array}$ & $\begin{array}{l}+ \text { Good localization } \\
\text { precision } \\
\text { - Requires synchronization } \\
\text { between transmitter (s) and } \\
\text { receiver (s) }\end{array}$ \\
\hline TDOA & $\begin{array}{l}\text { Distance } \\
\text { estimation based } \\
\text { on differences in } \\
\text { signal arrival } \\
\text { times }\end{array}$ & $\begin{array}{l}+ \text { Does not } \text { require } \\
\text { transmitter / } \\
\text { synchronization) } \\
\text { - Requires synchronization } \\
\text { of base stations }\end{array}$ \\
\hline RTT & $\begin{array}{l}\text { Distance } \\
\text { estimation based } \\
\text { on time } \\
\text { propagation of a } \\
\text { round trip } \\
\end{array}$ & $\begin{array}{l}+ \text { Does not require } \\
\text { transmitter / receiver } \\
\text { synchronization) } \\
-\quad \text { Requires high clock } \\
\text { accuracy }\end{array}$ \\
\hline $\mathrm{AOA}$ & $\begin{array}{l}\text { Position } \\
\text { estimation by } \\
\text { cross-checking } \\
\text { information on } \\
\text { angles of arrival }\end{array}$ & $\begin{array}{l}\text { + Position calculation with } \\
\text { a small number of } \\
\text { measurements } \\
\text { - High cost of equipment } \\
\text { (antennas) } \\
\text { - Accuracy that degrades as } \\
\text { the distance increases }\end{array}$ \\
\hline POA & $\begin{array}{l}\text { Distance } \\
\text { estimation by the } \\
\text { signal arrival } \\
\text { phases }\end{array}$ & $\begin{array}{l}\text { - unsuitable in location for } \\
\text { many technologies, such as } \\
\text { WiFi or BT }\end{array}$ \\
\hline RSSI & $\begin{array}{l}\text { Distance } \\
\text { estimation based } \\
\text { on the signal } \\
\text { strength } \\
\text { attenuation }\end{array}$ & $\begin{array}{l}\text { + Inexpensive and easily } \\
\text { measured by most radio } \\
\text { equipment } \\
\text { - High variance and low } \\
\text { consistency }\end{array}$ \\
\hline
\end{tabular}
with the main advantages and disadvantages in the Table 1.

Table 1. Indoor Localization metrics

\section{DESIGNING AN IOT INDOOR POSITIONING SYSTEM}

\subsection{IoT solutions architecture}

Given the rapid development of the IoT, it became necessary to have a reference architecture that would standardize the design 
of systems and promote interoperability and communication between the different ecosystems of the IoT (Lin et al., 2018). In term of technical implementation most of IoT solutions are based on three main components:

The end-devices that generate the data and send it to the gateway. The gateway that relays the data to the cloud, and the cloud platform. Gateways can also be called edge nodes and can inherit part of the computing functionalities for local data processing.

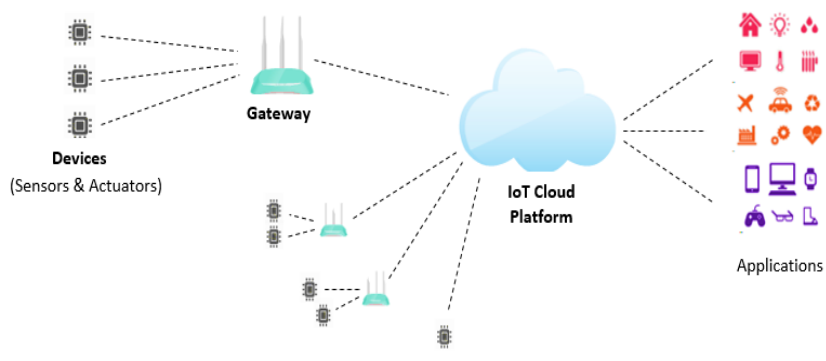

Figure 4. IoT solutions architecture

The IoT platform is a technical platform that collects data, monitors, supervises and controls connected objects. The IoT software platform embeds an intelligence that offers multiple possibilities. Measurements can be stored for the purpose of creating a history allowing prediction, management actions and providing a layer of intelligence (alerts, advice, etc.).

Four interactions models are defined between IoT actors:

Communication between objects: this model is based on wireless communication between two objects. Information is transmitted through the integration of wireless communication technology.

The communication of objects to the cloud: in this model, the data collected by the sensors is send to service platforms via a network. This includes radio communications (cellular, Bluetooth, WiFi, LoRaWAN ...) and light (the Li-Fi protocol uses the frequencies of the light spectrum to transmit information). This type of communication is used in hospital environments because it avoids the nuisance caused by radio communications such as WiFi.

Communication of objects to a gateway: this model is based on an intermediary that makes the link between the sensors and the applications in the cloud.

From objects to back-end data sharing: the purpose of this model is to enable data sharing between service providers. It is based on the "programmable web" concept. Manufacturers set up an API allowing the exploitation of data aggregated by other manufacturers.

\subsection{Positioning Approaches:}

Depending on where the measurements reside for position calculation, the positioning process is classified mainly in two approaches: Device centric positioning and Network centric positioning.

In case of device centric positioning approach the IoT end devices communicate, exchange information with their neighbours and make the location-related measurements.

In Network Centric Positioning approach when the entire position determination is carried out at other equipment independently of the devices. Each architecture requires different resources, communication scenarios, and privacy parameters. Security preserving is easier to be achieved in a device centric approach than in a network centric approach as the device would not reveal its position to the network (Mendoza-Silva et al., 2019; Salem et al., 2012).

\subsection{Position Estimation in IoT:}

Localization methods and algorithms use measurements to determine information on mobile terminal position. Two types of location information are possible: an area where the mobile terminal is located or the coordinates of mobile terminal position. The first represents a location by zone around a known point or zoning. The second is a localization by positioning which is divided into five main approaches: proximity, triangulation, multilateration, pedestrian dead reckoning and fingerprinting.

The positioning systems based on fingerprinting technique are the most developed in the last advanced researches (Basri and Elkhadimi, 2016; Oguntala et al., 2018) and produce the most accurate position estimation in indoor environments, due to the improved performances and the low cost of the required infrastructure. The process is mainly described in two phases: offline phase or training phase where we collect the measurements of signal characteristic to construct the radio map, then the online phase or positioning phase, in this phase a positioning algorithm is implemented to match online measurements with pre-recorded radio map and estimate the target position. In figure 5 the principle of fingerprinting technique is explained for a WiFi positioning system based on RSSI measurements received from different access points.

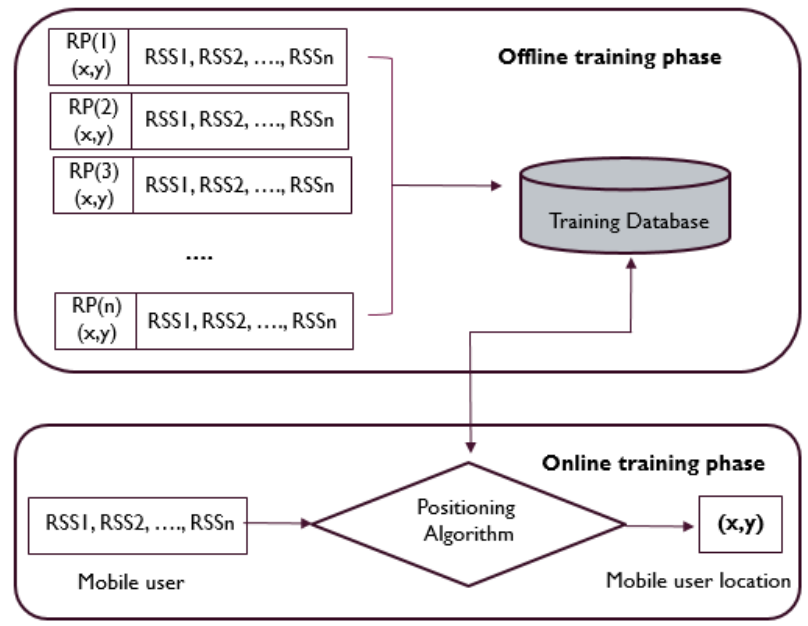

Figure 5. Fingerprinting based positioning

The localization algorithms may be based on deterministic approach, probabilistic approach, machine learning approaches or their combinations.

\subsubsection{Deterministic and Probabilistic Algorithms}

Deterministic positioning algorithm stores the values of RF metrics from each AP in the database, and utilizes real time matching of fingerprint data. Whereas the probabilistic approaches exploit additional information than simple values and need to compute and store the probability distribution model of each grid point in the radio map (Mendoza-Silva et al., 2019).

The major advantages of the deterministic methods are their ease of implementation. However, the probabilistic approaches are generally believed to be more effective because of their capability of dealing with signal strength variation and environment changers (Dardari et al., 2015). The main drawback 
is that it requires a large storage size which increases the time required to build the radio map.

\subsubsection{Machine Learning Algorithms}

Recent advanced fingerprint-based localization systems uses methods and techniques from machine learning for finding the optimal estimate of position (Cheng et al., 2016). The machine learning algorithms can be used for classification or regressions. In classification the machine learning algorithm learns to classify the data, and the output variable takes class labels, while in regression problems the algorithm predicts continuous values by learning from the training variables. In addition to position estimation process, machine learning algorithm can be applied to improve real time location service accuracy and reliability of the measurements. The notable algorithms for classification are: Decision trees, Principal Component Analysis (PCA), Naïve Bayes, K nearest Neighbours, Linear SVC (Support Vector Classifier) etc. And most of the practical machine learning approaches used for regression problems include: Support Vector Machine (SVM), Linear regression, Regression Trees (e.g Random Forest) etc.

The Naïve Bayes classifier has been widely used in machine learning and data mining and can achieve good performances (Mir et al., 2016). It is based on a solid mathematical theory. Naïve Bayes naïvely assumes that all the features are independent of each other. In our case the class node $\mathrm{C}$ represents the location and the features node RSSI is the received signal strength from access points.

In (Samaha et al., 2016), authors implemented in a WiFi indoor localization system multiple machine learning approaches and utilized the PCA algorithm to reduce the computation time and improve the performance. The system reduced the computation time by $70 \%$ when using Random Forest classifier in static mode and by $30 \%$ when using KNN in the dynamic mode.

The use of ML approaches in offline phase and online phase or the combination of multiple approaches can provide good performances. In (Zhang et al., 2017), authors introduced the PCA algorithm to reduce the dimension data in offline phase and grid search-based Kernel SVM is used in online phase to estimate the unknown location.

As we have illustrated in the following figure, machine learning can be exploited to determine target position and to improve system performance. Machine learning algorithms are implemented in data acquisition, classification, sensor fusion, signal correction, position estimation and output data analysis.

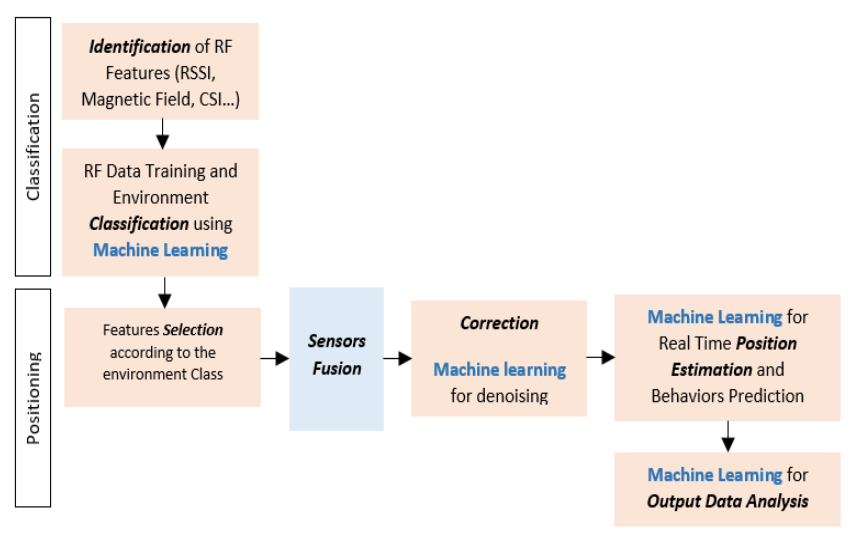

Figure 6. Machine learning applications in IoT positioning system

\subsection{IoT System parameters:}

The internet of things is thus a revolutionary paradigm that aims to enable many new applications. But it also raises a lot of challenges, and multiple factors could affect the localization accuracy.

IoT system parameters allow to assess the quality and the efficiency of the system, and a compromise between them should be considered while designing an IoT positioning system (Khelifi et al., 2019).

\subsubsection{Topology:}

The topology corresponds to the logical architecture of a network, defining the links between the objects of the network and the possible hierarchy between them.

In a Mesh topology, an object - called a node - is connected to one or more other nodes of the same network. This forms a mesh in which transmitted data is potentially relayed by several nodes before reaching its destination.

In star topology the objects are connected to a gateway that ensures communication between the nodes of the network as can be seen in the figure below. This type of topology makes it easy to add or remove nodes without impacting the rest of the network. In addition, all network intelligence is concentrated on a single node which makes it easier to manage the network. However, if the hub has a technical problem, then the entire network is down. In Indoor environment, mesh networks provide better coverage and better positioning accuracy than star networks, where some objects fail to connect to the gateway due to distance or noise and surrounding obstacles.
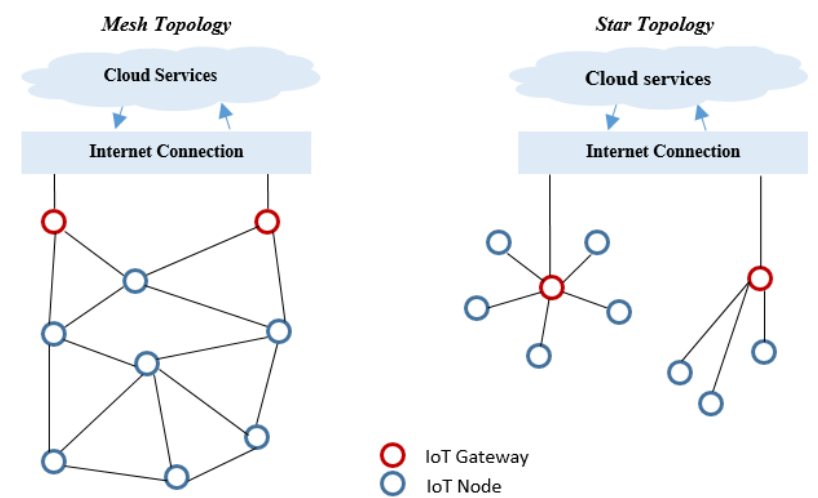

Figure 7. IoT mesh and star network topology

\subsubsection{The communication range:}

The communication range is the maximum distance at which a receiver can decode the signal. The range of a signal depends on both the value of the maximum transmitted power provided by the protocol and the physical environment. Generally, radio communications allow very long distances to be reached. In case of other technologies, a set of repeaters are deployed to relay the signal and overcome the coverage limitations. Therefore, depending on the distance between the object and the gateway, it will be obvious that some protocols will be more suitable than others.

\subsubsection{Bitrate:}

The bitrate measures the speed and the quantity of the data to be sent. The bitrate is mainly limited by the signal modulation and the frequency bandwidth: the wider the band, the higher is the bitrate.

The higher the amount of data that can be exchanged between devices, a more accurate localization can take place. 


\subsubsection{Hardware constraints}

IoT devices has several hardware resources constraints such as low battery capacity, RAM/Flash memory, CPU, power consumption, energy management and limited ability of storing, processing, and transporting data.

\subsubsection{Integrity}

Integrity is a mechanism ensuring that data is not: falsified, modified, altered, or deleted by an unauthorized entity. In most cases, this service is performed using hash functions with data signature properties.

\subsubsection{Interoperability}

The Internet of Things is a broad concept that encompasses different areas using different systems, architectures, and hardware. Therefore, an object must be able to interact and collaborate with other heterogeneous objects.

\subsubsection{Autonomy}

Autonomy is the ability of an object to control itself. It represents the properties of an entity that is capable of functioning independently and without being controlled from the outside. Indeed, each object has an identity representing it, can freely collect, analyse, process, generate and exchange information. $\mathrm{He}$ can also make decisions without any human intervention.

\subsection{IoT Challenges:}

The internet of things is thus a revolutionary paradigm that aims to enable many new applications. But it also raises a lot of challenges.

IoT touches on all areas of knowledge construction, businesses management and administrations through educational and hyperurban technologies, it transforms our localized relationship into a globalized relationship by going beyond geographic borders. These technological, societal, and human changes raise many challenges in research, creativity and innovation.

\subsubsection{Environmental challenges}

The constant increase of connected objects has multiple impacts on the environment. It can be translated by an increase of electronic waste, its recycling and energy consumption. This is a major challenge that is attracting more and more governments to study the impact of connected objects on global warming and their consequences on populations.

\subsubsection{Privacy}

Given the emergence of IoT technologies in all areas of daily life, this raises questions about IoT security (Lin et al., 2017). It must be thought out from 3 complementary angles: technological, human and systemic:

-Technology protection concerns the security of data, communications, network infrastructures and their functionalities.

-The protection of individuals concerns the protection of user's privacy to avoid litigation possibly caused by IoT.

-The protection of interconnected systems hosting IoT objects.

\subsubsection{Energy Consumption}

Several factors related to the programming of the IoT device will affect the energy consumption. Whenever possible it is appropriate to decrease the sensor sampling frequency or limit the use of actuators.

Energy consumption of a node is related to the operating mode of radio components. The stages of communication (transmissions and receptions, listening to the channel, duration of node activity) must therefore be controlled in order to conserve energy. It is possible to reduce power consumption on each node by controlling power transmission. Increasing the transmitted power improves the radio range of nodes, but also increases the risk of interference between them. The operator must therefore find a compromise between the number of deployed nodes, their hardware resources, connectivity, and transmitted power (Iqbal et al., 2017).

\section{CONCLUSION}

This paper presented an overview of indoor positioning systems implemented in IoT networks, domain applications, techniques of localization and communications based on recent researches. There is not one absolute solution for IoT Location Services. Each of the introduced technologies and approaches must be evaluated in the context of the target application goal, environment conditions, and system parameters. On the other hand we have highlighted how to exploit the use of machine learning algorithms, to improve system performances in term of data acquisition, classification, data fusion, denoising, and position estimation in order to provide more accurate system. In our future Work we aim to concept an hybrid system for real time location service, based on indoor environment classification and internetworking of different wireless positioning systems.

\section{REFERENCES}

Basri, C., El Khadimi, A. (2016, September). Survey on indoor localization system and recent advances of WIFI fingerprinting technique. In 2016 5th International Conference on Multimedia Computing and Systems (ICMCS) (pp. 253-259). IEEE.

Chen, G., Meng, X., Wang, Y., Zhang, Y., Tian, P., Yang, H. (2015). Integrated WiFi/PDR/Smartphone using an unscented kalman filter algorithm for 3D indoor localization. Sensors, 15(9), 24595-24614.

Cheng, Y. K., Chou, H. J., Chang, R. Y. (2016, May). Machinelearning indoor localization with access point selection and signal strength reconstruction. In 2016 IEEE 83rd Vehicular Technology Conference (VTC Spring) (pp. 1-5). IEEE.

Ebner, F., Fetzer, T., Deinzer, F., Grzegorzek, M. (2017). On Wi$\mathrm{Fi}$ model optimizations for smartphone-based indoor localization. ISPRS International Journal of GeoInformation, 6(8), 233.

Dardari, D., Closas, P., Djurić, P. M. (2015). Indoor tracking: Theory, methods, and technologies. IEEE Transactions on Vehicular Technology, 64(4), 1263-1278.

De Angelis, G., De Angelis, A., Pasku, V., Moschitta, A., \& Carbone, P. (2015, September). A hybrid outdoor/indoor Positioning System for IoT applications. In 2015 IEEE International Symposium on Systems Engineering (ISSE) (pp. 1$6)$.

Figueiredo e Silva, P., Kaseva, V., \& Lohan, E. S. (2018) Wireless positioning in IoT: A look at current and future trends. Sensors, 18(8), 2470.

Friis, H. T. (1946). A note on a simple transmission formula. Proceedings of the IRE, 34(5), 254-256.

Hossain, M. S., Muhammad, G., Alamri, A. (2019). Smart healthcare monitoring: A voice pathology detection paradigm for smart cities. Multimedia Systems, 25(5), 565-575. 
Iqbal, J., Khan, M., Talha, M., Farman, H., Jan, B., Muhammad, A., and Khattak, H. A. (2018). A generic Internet of Things architecture for controlling electrical energy consumption in smart homes. Sustainable cities and society, 43, 443-450.

Kamal, M., Aljohani, A., Alanazi, E. (2020). IoT meets COVID19: Status, Challenges, and Opportunities. arXiv preprint arXiv:2007.12268.

Khelifi, F., Bradai, A., Benslimane, A., Rawat, P., \& Atri, M. (2019). A survey of localization systems in internet of things. Mobile Networks and Applications, 24(3), 761-785.

Kok, M., Hol, J. D., Schön, T. B. (2017). Using inertial sensors for position and orientation estimation. arXiv preprint arXiv:1704.06053.

Li, Z., Liu, C., Gao, J., Li, X. (2016). An improved WiFi/PDR integrated system using an adaptive and robust filter for indoor localization. ISPRS International Journal of GeoInformation, 5(12), 224.

Lin, J., Yu, W., Zhang, N., Yang, X., Zhang, H., Zhao, W. (2017). A survey on internet of things: Architecture, enabling technologies, security and privacy, and applications. IEEE Internet of Things Journal, 4(5), 1125-1142.

Mendoza-Silva, G. M., Torres-Sospedra, J., Huerta, J. (2019). A meta-review of indoor positioning systems. Sensors, 19(20), 4507.

Nasiri, H., Nasehi, S., \& Goudarzi, M. (2019). Evaluation of distributed stream processing frameworks for IoT applications in Smart Cities. Journal of Big Data, 6(1), 52.

Oguntala, G., Abd-Alhameed, R., Jones, S., Noras, J., Patwary, M., Rodriguez, J. (2018). Indoor location identification technologies for real-time IoT-based applications: An inclusive survey. Computer Science Review, 30, 55-79.

Sadowski, S., Spachos, P. (2018). Rssi-based indoor localization with the internet of things. IEEE Access, 6, 30149-30161.

Saeed, N., Bader, A., Al-Naffouri, T. Y., Alouini, M. S. (2020). When Wireless Communication Faces COVID-19: Combating the Pandemic and Saving the Economy. arXiv preprint arXiv:2005.06637.

Salamah, A. H., Tamazin, M., Sharkas, M. A., \& Khedr, M. (2016, October). An enhanced WiFi indoor localization system based on machine learning. In 2016 International Conference on Indoor Positioning and Indoor Navigation (IPIN) (pp. 1-8). IEEE.

Salem, M., Ruppel, P., Bareth, U., Küpper, A. (2012, December). $\mathrm{X}$-centric positioning: A combination of device-centric and multi-rat network-centric positioning approaches in NGN. In 2012 IEEE Globecom Workshops (pp. 1741-1746). IEEE.

Sharma, K., Londhe, D. D. (2018, April). Human Safety Devices Using IoT and Machine Learning: A Review. In 2018 3rd International Conference for Convergence in Technology (I2CT) (pp. 1-7). IEEE.

Turgut, Z., Aydin, G. Z. G., Sertbas, A. (2016). Indoor localization techniques for smart building environment. Procedia computer science, 83, 1176-1181.
Yu, J., Na, Z., Liu, X., Deng, Z. (2019). WiFi/PDR-integrated indoor localization using unconstrained smartphones. EURASIP Journal on Wireless Communications and Networking, 2019(1), $1-13$.

Zhang, L., Li, Y., Gu, Y., Yang, W. (2017). An efficient machine learning approach for indoor localization. China Communications, 14(11), 141-150. 